\title{
A study of adatom ripening on an Al (111) surface with machine learning force fields
}

\author{
V. Botu, ${ }^{1, \text { a) }}$ J. Chapman, ${ }^{1}$ and R. Ramprasad ${ }^{1}$ \\ University of Connecticut, Storrs, CT 06269
}

Surface phenomena are increasingly becoming important in exploring nanoscale materials growth and characterization. Consequently, the need for atomistic based simulations is increasing. Recently, we proposed a machine learning approach, known as AGNI, that allows fast and quantum mechanical accurate atomic force predictions given an atom's neighborhood environment. Here, we make use of such force fields to study and characterize the nanoscale diffusion and growth processes occurring on an Al (111) surface. In particular we focus on the adatom ripening phenomena, confirming past experimental findings, wherein a low and high temperature growth regime were observed using entirely molecular dynamics simulations.

As the fabrication of materials continually progresses towards the atomic-scale, an interest in layer by layer growth methods (such as molecular-beam epitaxy or atomic layer deposition), in micro-electronics, catalysis, or biomedical applications, has risen tremendously. ${ }^{1-6}$ The high degree of control offered allows for a subnanometer scale precision in the morphological structure of the materials grown. Consequently, the need to better understand and characterize such growth processes, at the atomic level, has emerged.

Towards this cause, the advent of first-principles (also known as ab initio) based in silico models has been instrumental. Methods such as density functional theory, along with harmonic transition state theory, are now commonly used to (i) map out the energetics for the constitutive elementary reaction pathways, and (ii) then, rely on coarser stochastic approaches (e.g. kinetic Monte Carlo), to spatially and temporally evolve the state of a system, thus, helping unravel the complex atomistic growth phenomena at significantly larger length and time scales. ${ }^{7-9}$ Nevertheless, building a complete catalog of reaction pathways a priori is often challenging and nontrivial for low symmetry systems. An alternative, and more natural, formalism is to use molecular dynamics simulations, whereby the temporal state of an atomistic system is evolved by solving Newton's equations of motion. The key ingredient required for such methods is a description of the forces between the interacting atoms. Two methods - quantum mechanics or semi-empirical potentials, allow access to these forces. Unfortunately, the formidable computational cost of quantum mechanical methods restricts the time and length scales one can consider, while semi-empirical approaches provide a cheaper alternative but often lack the versatility and accuracy of quantum mechanical interactions. If pathways to accelerate $a b$ initio methods whilst retaining accuracy existed, they would be highly desired.

Off late, the prominence of machine learning methods, when used in tandem with quantum mechanical generated data, has demonstrated to be a reasonable trade-off between the cost, accuracy, and versatility issues facing

${ }^{a)}$ Electronic mail: botuvenkatesh@gmail.com current methods. ${ }^{10-12}$ Recently, we introduced one such framework, wherein, by encoding an atom's neighborhood environment numerically (its fingerprint), a mapping to the vectorial force components it experiences can be established. ${ }^{13-15}$ Such force based interpolative methods have been shown to be able to retain quantum mechanical accurate force predictions, with errors $<0.05$ $\mathrm{eV} / \AA$. Further, this high fidelity framework now comes at a fraction of the cost compared to quantum mechanical methods, up to six orders of magnitude faster. This allows us to explore long time scale dynamical behavior, as done in this work. The premise of mapping an atomic property (such as forces) and not the global potential energy for a configuration of atoms, as done by a majority of methods, allows this class of force fields to be more adaptive and generalizable for varying atomic environments. Consequently, the force field is referred to as AGNI - an adaptive, generalizable, and neighborhood informed methodology. For an extensive review on the force field itself, its scope and construction workflow, we refer the readers to our recent manuscript. ${ }^{15}$

This letter is intended to demonstrate the use of AGNI force fields in exploring nanoscale growth phenomena. In particular, we study the ripening of adatoms, whereby individual atoms cluster together to form larger island features, that occurs during the layer by layer growth process for an $\mathrm{Al}$ (111) surface, using true molecular dynamics (MD) simulations. This goes well beyond our previous work where we studied the dynamical behavior of a single adatom on the same surface. ${ }^{14}$ The (111) surface was chosen in particular as the barriers for the elementary processes allow for the ripening phenomena to be explored in time scales achievable with conventional MD simulations. The remainder of the letter is structured as follows. First, a brief review on the construction of AGNI force fields is provided. The force field is then validated by computing energetic barriers for elementary reaction pathways encountered on the surface. We then perform long time scale MD simulations exploring the ripening process as a function of two important parameters, time and temperature.

The construction of an accurate AGNI force field consists of 4 primary steps: (i) generating a diverse dataset of quantum mechanical reference atomic environments 


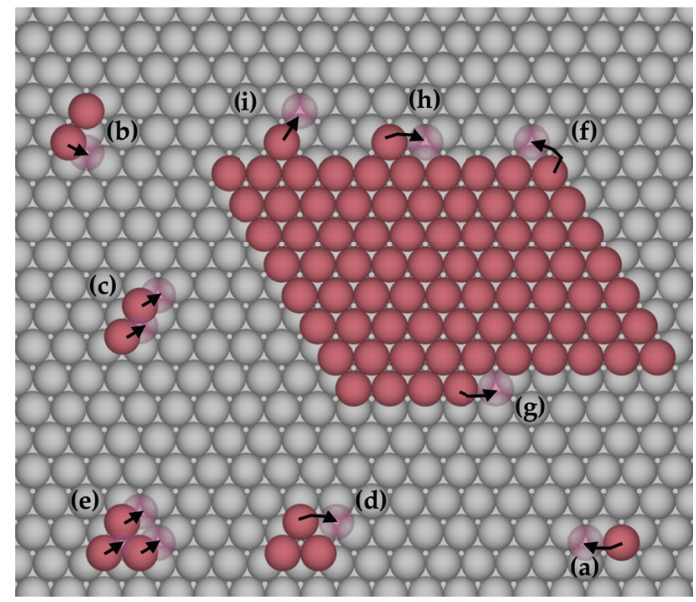

FIG. 1. Elementary reaction pathways of monomer, dimer, trimer, and other island features on an $\mathrm{Al}$ (111) surface that lead to the ripening phenomena. Grey and red colored atoms correspond to the surface atoms and adatoms, respectively.

TABLE I. Activation energy barriers for reaction pathways plotted in Figure 1, computed with AGNI ( $\mathrm{E}_{a}^{A G N I}$ ) and DFT. $\mathrm{E}_{a}^{A G N I}$ were obtained by integrating the forces. $\mathrm{E}_{a}^{D F T}$ were computed using the climbing-image nudged elastic band method, or sourced from literature as indicated by *.16,17

\begin{tabular}{lcc}
\hline \hline \multicolumn{1}{c}{ Pathway } & $\mathrm{E}_{a}^{A G N I}$ & $\mathrm{E}_{a}^{D F T}$ \\
\hline (a) Monomer hopping & 0.05 & 0.04 \\
(b) Dimer rotation & 0.12 & 0.11 \\
(c) Dimer translation & 0.13 & 0.07 \\
(d) Trimer translation & 0.19 & 0.21 \\
(e) Trimer rotation & 0.19 & 0.24 \\
(f) Corner evaporation & 0.71 & $0.60^{*}$ \\
(g) Kink evaporation & 0.67 & $0.65^{*}$ \\
(h) Edge diffusion & 0.48 & $0.45^{*}$ \\
(i) Edge evaporation & 0.91 & $0.80^{*}$ \\
\hline
\end{tabular}

and forces, (ii) choosing a numerical representation for the atomic environments, (iii) down selecting a representative set of training atomic environments, and (iv) identifying a learning method to map the environment to the force. A more detailed account of this workflow is provided elsewhere. ${ }^{13-15}$ We begin by compiling periodical and non-periodical atomic configurations of $\mathrm{Al}$, e.g., bulk, surfaces, clusters, defects, etc. For each configuration, ab initio based MD simulations, based on density functional theory (DFT), were performed at multiple temperatures to compile a diverse set of equilibrium and non-equilibrium atomic environments, along with their corresponding vectorial force components. All the DFT calculations were run using the VASP code at a PBE level of theory. ${ }^{18-21}$ Each atom and its environment is then encoded in a numerical manner using a directional dependent representation. The exact functional form is given below as

$$
V_{i}^{u}(\eta)=\sum_{j \neq i} \frac{r_{i j}^{u}}{r_{i j}} \cdot e^{-\left(\frac{r_{i j}}{\eta}\right)^{2}} \cdot f_{d}\left(r_{i j}\right)
$$

Here, $r_{i j}$ is the distance between atoms $i$ and $j\left(\left\|\mathbf{r}_{j}-\mathbf{r}_{i}\right\|\right)$, while $r_{i j}^{u}$ is a scalar projection of this distance along a direction $\hat{u} . \quad \eta$ is the Gaussian function width. Multiple such $\eta$ values are needed to accurately describe the environment around an atom. Here, $8 \eta$ values were sampled on a logarithmic grid between $[0.8 \AA, 16 \AA]$. $f_{d}\left(r_{i j}\right)=0.5\left[\cos \left(\frac{\pi r_{i j}}{R_{c}}\right)+1\right]$, is a damping function for atoms within the cutoff distance $\left(R_{c}\right)$, and is zero elsewhere. An $R_{c}$ of $8 \AA$ is used in the present work. From all the atomic environments compiled, a subset of these are chosen with the help of dimensionality reduction methods and sampling techniques, to ensure that the information content within these environments is diverse. Here 3000 such environments are chosen.

To map an atomic fingerprint $\left(V_{i}^{u}\right)$ to the individual force component $\left(F_{i}^{u}\right)$ we rely on a non-linear kernel ridge regression framework,

$$
F_{i}^{u}=\sum_{t}^{N_{t}} \alpha_{t} \cdot \exp \left[-\frac{\left(d_{i, t}^{u}\right)^{2}}{2 l^{2}}\right] .
$$

Here, $t$ labels each reference atomic environment, and $V_{t}^{u}(\eta)$ is its corresponding fingerprint. $N_{t}$ is the total number of reference environments considered. $d_{i, t}^{u}=$ $\left\|V_{i}^{u}(\eta)-V_{t}^{u}(\eta)\right\|$, is the Euclidean distance between any two atomic fingerprints. $\alpha_{t} \mathrm{~s}$ and $l$ are the weight coefficients and Gaussian length scale parameter, respectively, determined using standard machine learning crossvalidation practices. ${ }^{22}$ The constructed AGNI force field has a mean absolute prediction error $(\mathrm{MAE})<0.05$ $\mathrm{eV} / \AA$, on the order of expected chemical and numerical accuracy of the reference quantum mechanical calculations.

Moving on, a first step to the realization of the ripening process is to ensure that the elementary reactions occurring on the surface, such as translation, rotation and diffusion of adatoms, monomer, dimer, trimer and beyond, as well as processes corresponding to re-arrangement of islands, such as corner breaking, kink breaking, terrace diffusion, edge evaporation etc., as illustrated in Figure 1 , are correctly described. Here, we compute the reaction energy barriers for all pathways, (a)-(i), shown in Figure 1 using the AGNI force fields $\left(\mathrm{E}_{a}^{A G N I}\right)$. The barriers are reported in Table I. Given that AGNI force fields provide access to the forces only we compute the energies via thermodynamic integration of the forces. ${ }^{15}$ For comparison we report the corresponding DFT computed reaction barriers $\left(\mathrm{E}_{a}^{D F T}\right)$, using the climbing-image nudged elastic band method and those reported in the literature (highlighted by $*$ in Table I) ${ }^{16,17}$ The errors are within $5 \%$ of the DFT computed values. In principle, the accuracy can be improved upon by directly including these atomic 


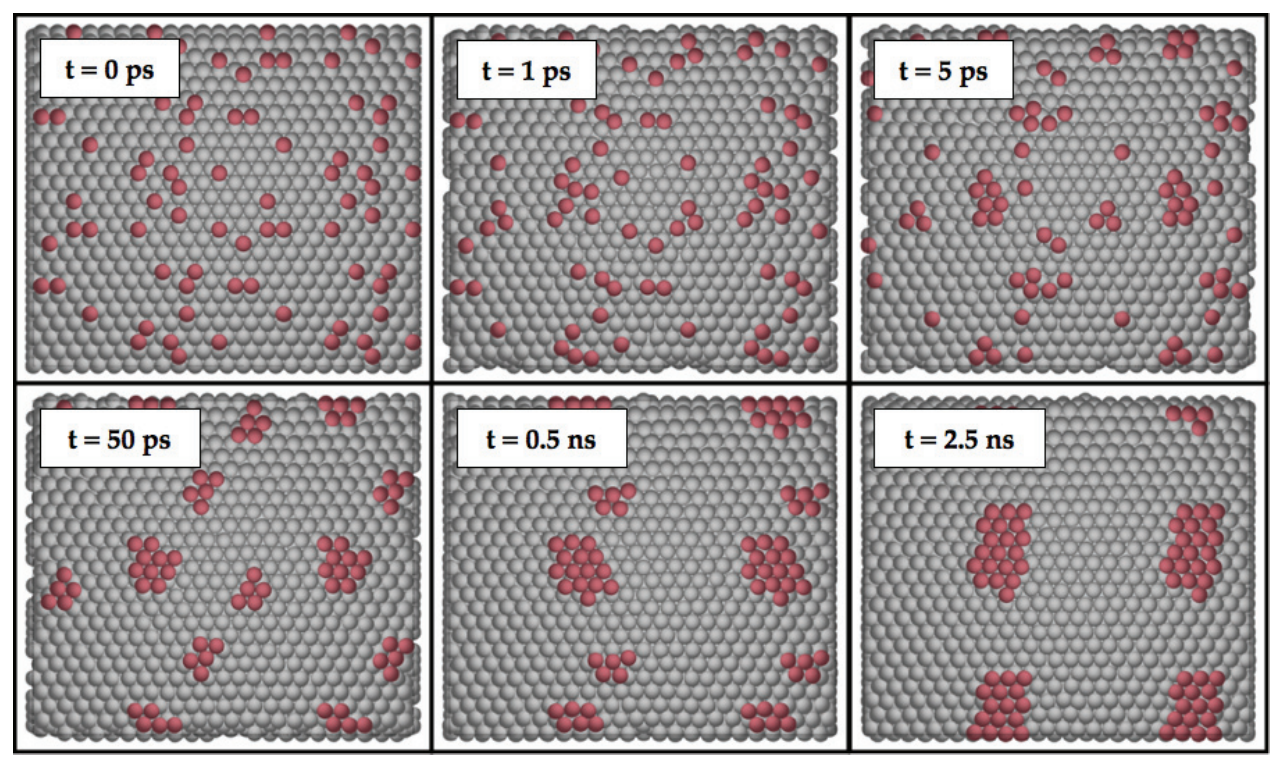

FIG. 2. Snapshots of the time-evolution of adatoms on Al (111) surface using constant temperature (300 K) molecular dynamics simulation. Adatoms were randomly distributed on the surface as shown at $\mathrm{t}=0 \mathrm{ps}, \theta=0.14$. The images shown are a $2 \times 2$ repeat of the unit cell. Grey and red colored atoms correspond to the surface atoms and adatoms, respectively.

environments during force field training, though such an undertaking is not considered here.

Having demonstrated an accurate description of the elementary processes we now study the ripening of adatoms on the $\mathrm{Al}$ (111) surface. To do so we construct a $35 \times$ $30 \AA^{2}$ asymmetric surface model, with 6 layers, where, the bottom layer is fixed in position and the remaining layers are considered during the dynamics. The adatoms are then randomly distributed on the free surface, and the concentration of adatoms is described by a coverage $(\theta)$, given as the ratio of adatoms on the surface to the maximum acceptable number. The dynamic simulations were performed in the canonical ensemble, with a timestep of $0.5 \mathrm{fs}$, using the popular LAMMPS MD code. ${ }^{23} \mathrm{~A}$ tarball containing the force field and LAMMPS input files is provided in the supplemental information..

We start by exploring the temporal evolution of a system with $\theta=0.14$ at $300 \mathrm{~K}$. Snapshots during the course of the dynamic simulation are illustrated in Figure 2, up to a few nanoseconds. The randomly distributed adatoms quickly (in a few picoseconds) form small islands with a density $\approx 4-5$ atoms, and once formed remain intact. This is consistent with past theoretical studies where dimers, trimers, and larger clusters were shown to be stable once formed and prefer to move in a concerted manner ${ }^{17,24-26}$. As island density increases, the mobility decreases, given the proportional number of bonds that need to be broken, significantly increasing the time between any relevant concerted displacements. Nevertheless, at $300 \mathrm{~K}$ the thermal energy is sufficient to overcome these barriers and the individual clusters ripen to form an island after $2 \mathrm{~ns}$. Also, the island formed is primarily $2 \mathrm{D}$, with no observable evaporation of adatoms (pathway (i) in Figure 1).
Temperature plays a critical role in the morphological shape and density of the islands formed. Past experimental studies, at $\theta=0.11$, by Busse et al. reveals distinct structural patterns for the islands formed in a temperature range of $50-300 \mathrm{~K}^{27,28}$ At low temperatures the lack of thermal energy results in hit and stick islands, that are formed by aggregation of nearby atoms to form several small clusters of islands, that transitions into compact islands as the temperature increases. To better understand the role of temperature, dynamical simulations between $50 \mathrm{~K}-300 \mathrm{~K}$ were performed, as shown in Figure 3 (top panel). At $100 \mathrm{~K}$, we observe several small islands that remain immobile once formed. At $200 \mathrm{~K}$, the islands grow in a dendritic fashion, whereby clusters of atoms group together with no significant rearrangement. The elementary processes, such as kink breaking, corner diffusion, etc., required to smoothen islands are activated only at high temperatures, and in the case of $\mathrm{Al}$ (111) beyond $250 \mathrm{~K}$. These findings are consistent with past kinetic Monte Carlo results for other metal surfaces, whereby, fractal patterns dominate at low temperature, which transition into dendritic patterns, and culminate as compact islands as the temperature increases. ${ }^{16,29,30}$ Note that the shape of islands observed in the MD simulations will be governed by the underlying reference theory used. It is known that a PBE level theory fails to correctly capture the anisotropy behavior of edge and corner diffusions, leading to triangular like rather than compact islands. ${ }^{30}$ Such deficiencies translate in our simulations as well. Nevertheless, the data-driven nature of AGNI force fields provides the flexibility of incorporating more accurate reference data to overcome such deficiencies.

Futher, in the case of an $\mathrm{Al}$ (111) surface Busse et al. experimentally observed two distinct growth regimes, as 

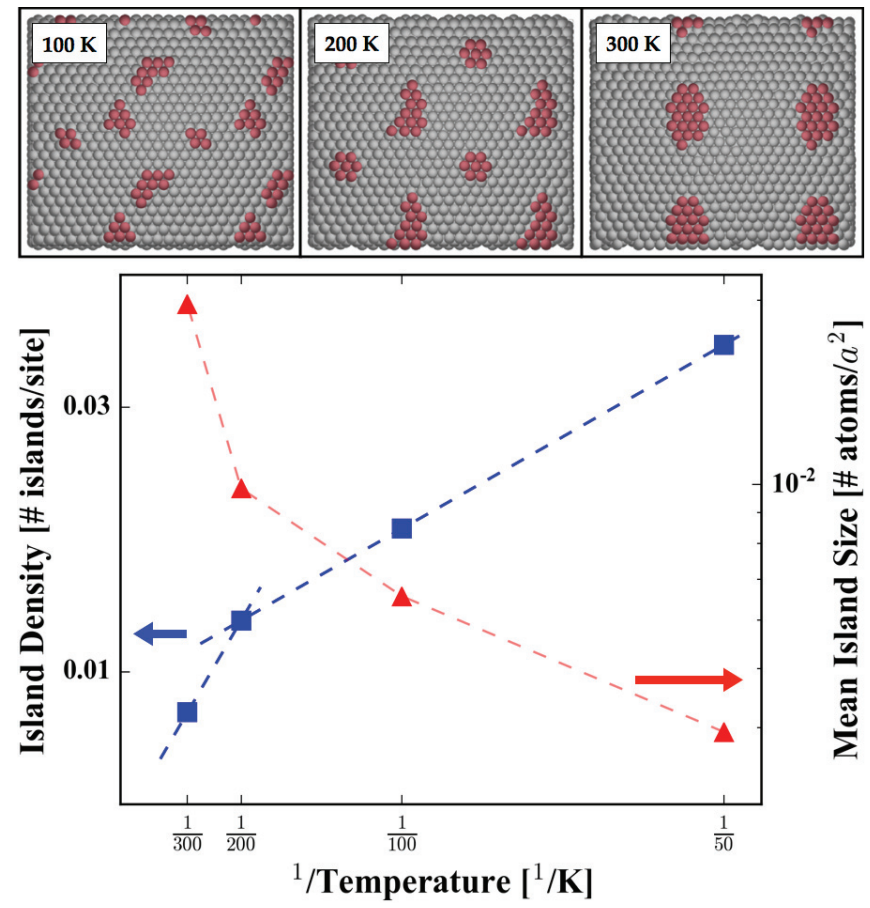

FIG. 3. Top Panel: Island ripening as a function of temperature. Shown here is a simulation at the end of $2.5 \mathrm{~ns}$ for 100 $\mathrm{K}, 200 \mathrm{~K}, 300 \mathrm{~K}$, at $\theta=0.14$. Grey and red colored atoms correspond to the surface atoms and adatoms, respectively. Bottom Panel: Island density and size as a function of temperature. Two scaling regimes are observed with a transition temperature of $\approx 200 \mathrm{~K}$.

measured by the change in island density and temperature $(\mathrm{T})$, one at $\mathrm{T}<200 \mathrm{~K}$ and one above $\mathrm{T}>200 \mathrm{~K}^{27}$. We undertake a similar such analysis for the structures generated by the molecular dynamics simulations (c.f., bottom panel of Figure 3). An identical transition, consistent with the past experimental results, is observed at $\mathrm{T} \approx 200 \mathrm{~K}$. For the two regimes we compute the activation energy by measuring the slope, resulting in values of $1.2 \mathrm{~K}^{-1}$ and $4.2 \mathrm{~K}^{-1}$. The consistent features observed (island shapes and growth regimes) suggests that the MD simulations undertaken with AGNI force fields drives the dynamical evolution in a manner consistent with both the thermodynamics and kinetics of the ripening process.

To summarize, in this work we demonstrated the use of machine learning force fields in studying the ripening phenomena of adatoms on an $\mathrm{Al}$ (111) surface, using MD simulations. Here, we confirm the two growth regimes observed experimentally in a temperature range of 50 $300 \mathrm{~K}$. At low temperatures the ripening phenomena is localized resulting in fractal like islands, and transitions into more compact islands as the temperature increases. This transition is observed at a temperature of $\approx 200$ $\mathrm{K}$, and is in excellent agreement with past experimental data. Clearly, the simulations undertaken by combining machine learning methods and quantum mechanical data demonstrates the fidelity in describing dynamical phenomena at larger length and time scales. The dependence on such hybrid methodologies will become increasingly more important, as we continually strive to push the envelope of atomistic modeling capabilities to more interesting materials phenomena, e.g., phase transformations or reactions on surfaces, all of which require going beyond a purely quantum mechanical description.

This work was supported financially by the Office of Naval Research (Grant No. N00014-14-1-0098). The authors would like to acknowledge K. B. Lipkowitz for helpful discussions, and S. K. Yadav and G. Pilania for reviewing the manuscript.

${ }^{1}$ R. W. Johnson, A. Hultqvist, and S. F. Bent, "A brief review of atomic layer deposition: from fundamentals to applications," Materials today 17, 236-246 (2014).

${ }^{2}$ S. M. George, "Atomic layer deposition: An overview," Chemical Reviews 110, 111-131 (2010).

${ }^{3}$ B. S. Lim, A. Rahtu, and R. G. Gordon, "Atomic layer deposition of transition metals," Nature materials 2, 749-754 (2003).

${ }^{4}$ M. A. Herman and H. Sitter, Molecular beam epitaxy: fundamentals and current status, Vol. 7 (Springer Science \& Business Media, 2012).

${ }^{5}$ B. R. Pamplin, Molecular beam epitaxy (Elsevier, 2013).

${ }^{6}$ Z. Zhang and M. G. Lagally, "Atomistic processes in the early stages of thin-film growth," Science 276, 377-383 (1997).

${ }^{7} \mathrm{~J}$. A. Elliott, "Novel approaches to multiscale modeling in materials science," Int. Mat. Rev. 56, 207 (2011).

${ }^{8} \mathrm{~F}$. Voter, "A method for accelerating the molecular dynamics simulation of infrequent events," J. Chem. Phys. 106, 4665 (1997).

${ }^{9}$ G. Pilania, P.-X. Gao, and R. Ramprasad, "Establishing the lamno3 surface phase diagram in an oxygen environment: An ab initio kinetic monte carlo simulation study," The Journal of Physical Chemistry C 116, 26349-26357 (2012).

${ }^{10}$ A. P. Bartok, M. C. Payne, R. Kondor, and G. Csanyi, "Gaussian approximation potentials: The accuracy of quantum mechanics without the electrons," Phys. Rev. Lett. 104, 136403 (2010).

${ }^{11} \mathrm{~J}$. Behler, "Neural network potential-energy surfaces in chemistry: a tool for large-scale simulations," Phys. Chem. Chem. Phys. 13, 17930 (2011).

${ }^{12}$ Z. Li, J. R. Kermode, and A. De Vita, "Molecular dynamics with on-the-fly machine learning of quantum-mechanical forces," Phys. Rev. Lett. 114, 096405 (2015).

${ }^{13} \mathrm{~V}$. Botu and R. Ramprasad, "Adaptive machine learning framework to accelerate ab initio molecular dynamics," Int. J. Quant. Chem. 115, 1074-1083 (2015).

${ }^{14} \mathrm{~V}$. Botu and R. Ramprasad, "Learning scheme to predict atomic forces and accelerate materials simulations," Phys. Rev. B 92, 094306 (2015).

${ }^{15}$ V. Botu, R. Batra, J. Chapman, and R. Ramprasad, "Machine learning force fields: Construction, validation and outlook," J. Phys. Chem. C, in review (2016).

${ }^{16}$ A. Bogicevic, J. Strömquist, and B. I. Lundqvist, "Lowsymmetry diffusion barriers in homoepitaxial growth of al(111)," Phys. Rev. Lett. 81, 637-640 (1998).

${ }^{17}$ A. Bogicevic, P. Hyldgaard, G. Wahnström, and B. I. Lundqvist, "Al dimer dynamics on al(111)," Phys. Rev. Lett. 81, 172-175 (1998).

${ }^{18}$ G. Kresse and J. Furthmuller, "Efficient iterative schemes for $a b$ initio total-energy calculations using a plane-wave basis set," Phys. Rev. B 54, 11169 (1996).

${ }^{19}$ G. Kresse and D. Joubert, "From ultrasoft pseudopotentials to the projector augmented-wave method," Phys. Rev. B 59, 1758 (1999).

${ }^{20}$ P. E. Blöchl, "Projector augmented-wave method," Phys. Rev. B 50, 17953 (1994).

${ }^{21}$ R. Car and M. Parrinello, "Unified approach for molecular dy- 
namics and density-functional theory," Phys. Rev. Lett. 55 2471-2474 (1985).

${ }^{22}$ T. Hastie, R. Tibshirani, and J. Friedman, The Elements of Statistical Learning: Data Mining, Inference, and Prediction, 2nd ed. (Springer, New York, 2009).

${ }^{23}$ S. Plimpton, "Fast parallel algorithms for short-range molecular dynamics," Journal of computational physics 117, 1-19 (1995).

${ }^{24} \mathrm{R}$. Stumpf and M. Scheffler, "Ab initio calculations of energies and self-diffusion on flat and stepped surfaces of al and their implications on crystal growth," Phys. Rev. B 53, 4958-4973 (1996).

${ }^{25}$ C. Chang, C. Wei, and S. Chen, "Structural and dynamical behavior of al trimer on al(111) surface," Surface Science 465, $65-75(2000)$.

${ }^{26}$ C. M. Chang, C. M. Wei, and S. P. Chen, "Self-diffusion of small clusters on fcc metal (111) surfaces," Phys. Rev. Lett. 85, 1044-1047 (2000).
${ }^{27}$ C. Busse, W. Langenkamp, C. Polop, A. Petersen, H. Hansen, U. Linke, P. J. Feibelman, and T. Michely, "Dimer binding energies on fcc(1 11 1) metal surfaces," Surface Science 539, L560 L566 (2003)

${ }^{28}$ C. Busse, C. Engin, H. Hansen, U. Linke, T. Michely, and H. M. Urbassek, "Adatom formation and atomic layer growth on al(1 1 1) by ion bombardment: experiments and molecular dynamics simulations," Surface Science 488, 346 - 366 (2001).

${ }^{29}$ C. Ratsch, P. Ruggerone, and M. Scheffler, "Density-functional theory of surface diffusion and epitaxial growth of metals," in Surface Diffusion, NATO ASI Series, Vol. 360, edited by M. Tringides (Springer US, 1997) pp. 83-101.

${ }^{30} \mathrm{~S}$. Ovesson, A. Bogicevic, and B. I. Lundqvist, "Origin of compact triangular islands in metal-on-metal growth," Phys. Rev. Lett. 83, 2608-2611 (1999). 
